\title{
Accurate skin cancer diagnosis based on convolutional neural
} networks

\author{
Amal G. Diab ${ }^{1}$, Nehal Fayez ${ }^{2}$, Mervat Mohamed El-Seddek ${ }^{1}$ \\ 'Department of Communications and Electronics Engineering, MISR Higher Institute for Engineering and Technology, Mansoura, Egypt \\ ${ }^{2}$ Department of Electronics and Communications Engineering, Faculty of Engineering, Mansoura University, Mansoura, Egypt
}

\begin{tabular}{l} 
Article Info \\
\hline Article history: \\
Received Nov 21, 2021 \\
Revised Dec 28, 2021 \\
Accepted Jan 17, 2022 \\
\hline
\end{tabular}

Keywords:

Computer aided diagnosis Convolutional neural network

Melanoma

Skin cancer

Support vector machine

\begin{abstract}
Although melanoma is not the most common type of skin cancer, it is supposed to extend to other areas of the body if not early diagnosed. Melanoma is the deadliest form of skin cancer and accounts for about $75 \%$ of deaths associated with skin cancer. The present study introduces an automated technique for skin cancer prediction, detection, and diagnosis including trending noninvasive and nonionizing techniques that combines deep learning methods to diagnose melanoma with high accuracy. Computer-aided diagnosis (CAD) using medical images is utilized to distinguish benign and malignant tumors, which can assist physicians in early identification of symptoms, thus lowering the mortality rate. The CAD system consists of four phases; detection of the region of interest (RoI), using data augmentation techniques, processing RoI using convolutional neural network $(\mathrm{CNN})$ to extract the most important features, and finally the extracted CNN features are input to a support vector machine (SVM) classifier to decode the two classes benign (B) and malignant (M). Two datasets, ISIC and CPTAC-CM, were utilized to train the CNNs. GoogleNet, ResNet-50, AlexNet, and VGG19 were investigated and compared. The accuracy of the proposed CAD system has reached $99.8 \%$ for ISIC database and $99.9 \%$ for CPTAC-CM database.
\end{abstract}

This is an open access article under the CC BY-SA license.

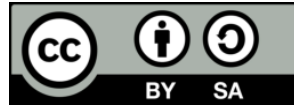

\section{Corresponding Author:}

Mervat Mohamed El-Seddek

Department of Communications and Electronics Engineering

MISR Higher Institute for Engineering and Technology

Mansoura, Egypt

Email: mervat.elseddek@ieee.org

\section{INTRODUCTION}

Skin cancer is characterized by irregular alterations in the skin's outer layer. It is one of the most widespread cancers in the world. According to the World Health Organization (WHO), skin cancer has been one of the most common forms of cancers and melanoma is one of the leading causes of death, particularly in USA, Australia, and New Zealand [1]. While the majority of skin cancer patients are cured, the disease remains a significant concern because of its rise pervasiveness [1]. The majority of skin tumors grow only internally and penetrate nearby tissues, but others, such as melanoma (pigment cell cancer), which is the most extraordinary type of skin malignant growth, can prevalence across the circulatory or lymphatic systems and penetrate the most remote parts for the human body [2]. Melanoma has the greatest risk of the various types of skin cancer [3]-[7]. Melanoma claimed the lives of 4740 men and 2490 women in 2019 [8]. The findings show that diagnosing melanoma in its early stages will dramatically decrease cancer-related mortality. Since diagnosing melanoma in an early phase is complex, it will be extremely beneficial to develop a procedure for accurate and early detection of melanoma [9]-[12]. The use of image recognition and machine vision to 
quickly distinguish patterns such as tumors from images decreases human error and improves detection time [13]-[19]. One of the most commonly used techniques of image analysis is neural networks. Convolutional neural networks (CNNs) represent a form of deep neural networks that are widely used in machine learning for medical applications [20]-[25]. An automated decision framework is suggested that involves various machine learning techniques to automatically identify melanoma skin lesions with high precision. In the present work, both the International Skin Imaging Collaboration (ISIC 2019) dataset [26]-[28] and the Clinical Proteomic Tumor Analysis Consortium Cutaneous Melanoma (CPTAC-CM) dataset have been utilized [29]. Figures 1 and 2 show examples from the two datasets. Four CNNs are utilized to recognize the common nevus from melanoma. To accomplish most elevated accuracy, the framework utilizes the accuracies acquired by each method separately. Each process adds a proportion of the final decision taken by the main system based on the accuracy obtained. The AlexNet, ResNet-50, GoogleNet and VGG19 were fine-tuned by utilizing transfer learning to diagnose skin lesions. In terms of the feature-based process, classification will be accomplished using support vector machine (SVM).

This paper aims to develop a framework for the early prediction of skin cancer utilizing CNN modeling, two datasets and data augmentation, to solve the following existing work's limitations: Building new CNN models necessitates a massive amount of data for modeling in order to prevent over-fitting. Furthermore, these methods are computationally costly, especially during the training process; and Advanced techniques must be created and develop new ideas to increase diagnostic accuracy.

The motivations and contributions of the study will be present as: Select a framework from four CNN models to enhance the current skin cancer classification models. This enhancement generates an application that appeals to the overall conditions; and propose a very accurate and fast computer-aided diagnosis (CAD) system, to leverage deep learning's benefits for image analysis and high performance. Because CNN models and frameworks may be retrained using a bespoke dataset for every use case, deep learning offers greater flexibility.

The proposed system has the following contributions: i) The proposed CAD system employs an automated optimal approach to isolate the region of interest (RoI) from the background in order to identify skin lesions; ii) Investigating and comparing different deep learning architectures for skin cancer detection (i.e., AlexNet, ResNet-50, GoogleNet and VGG19); iii) Improving the performance of deep learning training using data balancing and augmentation; and iv) Predicting skin cancer early with 100\% accuracy. This study will aid in the selection of the most appropriate and accurate model for skin cancer diagnosis based on the severity of the case critical patients either non-critical patient.

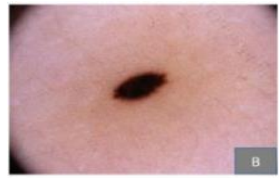

(a)

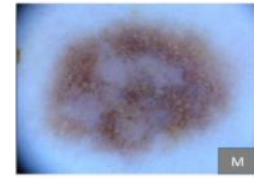

(b)

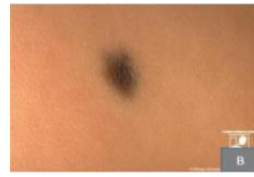

(a)

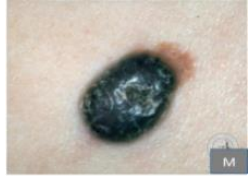

(b)

Figure 1. Examples from ISIC 2019 database [26][28], (a) benign and (b) malignant

Figure 2. Examples from CPTAC-CM database [29], (a) benign and (b) malignant

\section{RELATED RESEARCH}

Over the course of several years, numerous research works on skin cancer have been conducted. Some of these techniques have showed substantial prediction accuracy. Many researchers have used image segmentation techniques and machine learning classifiers like SVM, KELM, k-nearest neighbor (KNN), and FP-ANN to improve treatment for skin cancer detection.

To detect skin cancer early, $\mathrm{Li}$ and Shen [30] introduced a melanoma detection analysis of skin lesions. Two deep learning approaches ( 2 fully convolutional residual networks-FCRN) were proposed to address lesion segmentation, feature extraction, and classification of lesions. The obtained accuracy for lesion segmentation was 0.753. Kassem et al. [31] discussed the papers that have been accepted for publication within last five years in ScienceDirect, IEEE, and SpringerLink databases. This research identified the major challenges of assessing skin lesion segmentation and classification techniques, such like small datasets, ad hoc image selection, and racial bias. Hosny et al. [32], for example, used Alexnet with transfer learning to classify the challenging dataset ISIC2018. For lesion segmentation, they used a variety of techniques. The obtained accuracy was $92.99 \%$. Hosny et al. [33] used the challenging dataset ISIC2019 and proposed a CAD system for skin lesions. That dataset has several flaws, including imbalanced classes and unknown 
images. The bootstrap weighted classifier was combined utilizing a multiclass SVM by the authors. This classifier adjusted the weights based on the image class. The obtained accuracy was $98.70 \%$. Khan et al. [34] proposed a fully automated method for the segmentation and categorization of multiclass skin lesions that makes use of the most discriminant deep features. They segmented the image utilizing a novel Deep Saliency Segmentation technique that employs a ten-layer custom convolutional neural network (CNN). They used the kernel extreme learning machine (KELM) classifier to categorise skin lesions. On the ISBI 2016, ISBI 2017, ISIC 2018, and PH2 datasets, the accuracies for lesion segmentation were 95.3\%, 95.79\%, 92.69\%, and $98.70 \%$, respectively. The classification accuracy obtained on the HAM10000 dataset was $90.67 \%$. Abayomi-Alli et al. [35] proposed an improved data augmentation model for melanoma skin cancer detection. Their method for creating synthetic melanoma images was focused on data oversampling in a lower-dimensional nonlinear embedding manifold. The SqueezeNet deep learning model was trained using the augmented images. Results in the binary classification problem had shown enhanced melanoma detection accuracy of $92.18 \%$, sensitivity of $80.77 \%$, and specificity of $95.1 \%$. The paper is organized as follows; section 2 presents detailed descriptions of materials and methods, and section 3 contains experimental results and discussion, and finally section 4 gives the conclusions of the paper.

\section{MATERIALS AND METHOD}

The proposed melanoma deep learning framework consists of four processing stages as illustrated in Figure 3. First, The RoI is identified from the input image. Second, Raw input data images are augmented using ten cropping/flipping, then deep features are extracted from augmented images. Finally, classification is carried out in order to establish the skin cancer classes (benign and malignant) grading utilizing a support vector machine (SVM) classifier.

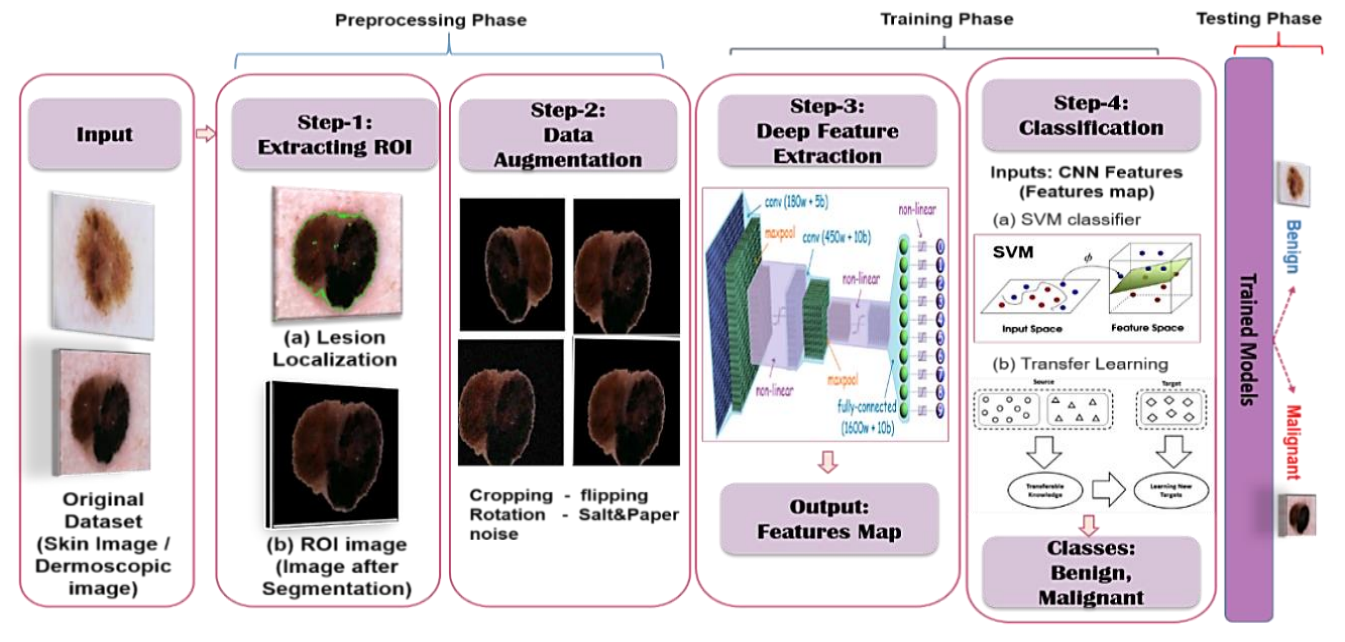

Figure 3. The proposed CAD scheme for skin cancer detection

\subsection{Collected database and data preparation}

The images utilized in the present work to train and validate the proposed techniques were taken from two separate free dermoscopic images datasets; the International Skin Imaging Collaboration (ISIC) 2019 database [26]-[28], and the clinical proteomic tumor analysis consortium cutaneous melanoma (CPTAC-CM) database obtained from the TCIA [29]. The ISIC is an international initiative to enhance early detection of melanoma. The ISIC Archive contains about 25,331 JPEG images of skin lesions. The ISIC 2019 was created specifically for research purposes to aid in the suggestion of new techniques that can be used as an assistive diagnostic tool of melanoma [26]-[28]. The TCIA is the archive for cancer imaging and associated material at the National Cancer Institute in the United States. It incorporates 30.9 million radiology photographs that provides data from 37,568 subjects. Data is divided into categories based on tumor type, with several categories containing either analytic observations or clinical evidence [29].

\subsection{Image segmentation}

One of the most crucial procedures in efficiently classifying lesions is segmenting the lesion from the surrounding skin [36]. Because there was a noticeable colour difference between the lesion and the skin, thresholding was an excellent choice for this job. As illustrated in Figure 4, a binary image was created with 
the contrast increased to improve segmentation. To validate the segmentation accuracy, the image is also subjected to a boundary tracing algorithm. Thresholding is a straightforward method of segmenting a digital image. It can be used with either a greyscale or a colour image. The basic principle underlying thresholding involves a parameter called "intensity value" or "brightness threshold," this parameter is selected in such a way that given an image $I[M, N]$, if $I[M, N] \geq$ brightness threshold, consequently $I[M, N]=1$, else $I[M, N]=0$. This is true if all we care about bright items against a dark background; however, if we are interested in dark objects against a light background, the equation would be reversed. The thresholding technique is also a computationally low-cost strategy that does not necessitate a large amount of computing time or power. The outcomes of segmenting the lesion with the specified algorithm are illustrated in Figure 4.

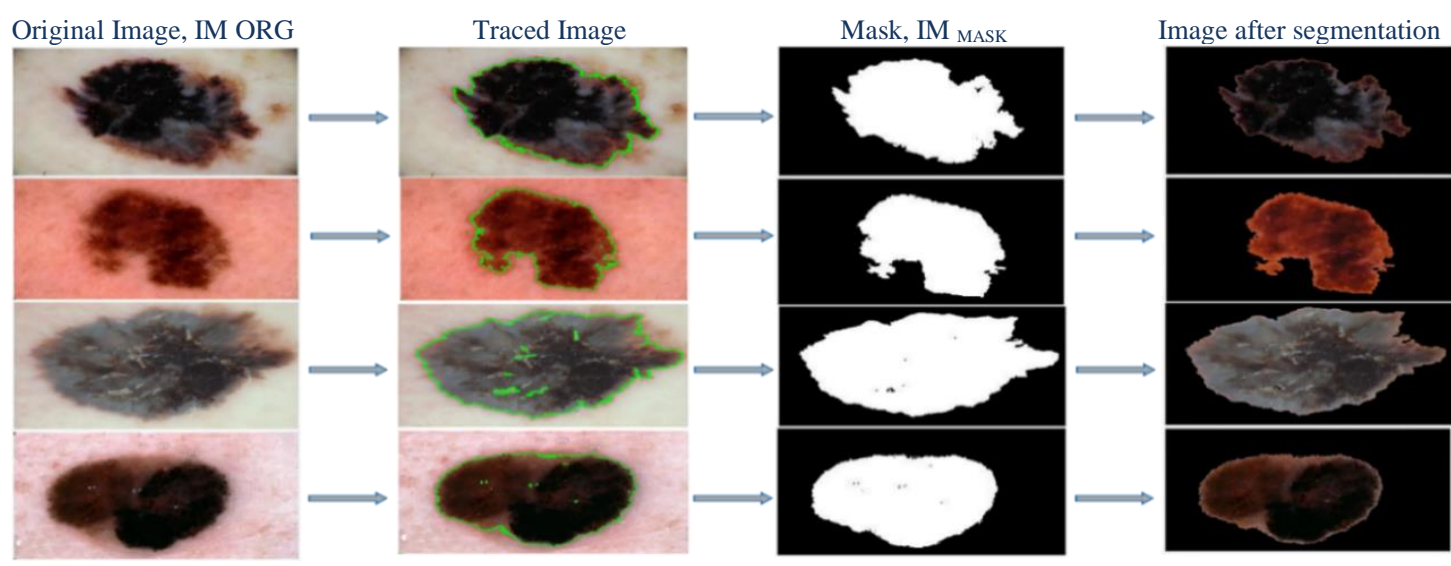

Figure 4. Extracting roi from the background

\subsection{Data augmentation and balancing}

The ISIC and CPTAC-CM databases are augmented to enhance the efficiency of the proposed deep learning framework, enhance the dataset size and prevent over-fitting. To start, the original image and the segmented image are resized from their original sizes to $250 \times 250$ to allow for high speed processing. We repeat the augmentation process described in [37]; five cropped images with dimensions of $224 \times 224$ are produced as follows: four images are cropped from each corner of the image and one image is cropped around the center of the image, so that the size of any cropped image is $224 \times 224$. Further, the five cropped images were flipped horizontally around the vertical axis to create a total of ten cropped images of size $224 \times 224$ as shown in Figure 5.

Since (i) the number of images per each grade for the ISIC and CPTAC-CM is unbalanced, and (ii) the size of the augmented data is ten time the size of the raw data, the proposed system aims to balance the data by arbitrarily choosing an equal certain number of images for each grade in order to prevent biasing the training results. This step is referred to as "data balancing". The detection rate for each class should ideally be near to or the same. We can conclude from the examples above that using a balanced dataset for a model results in higher accuracy models, higher precision, and a higher detection rate.

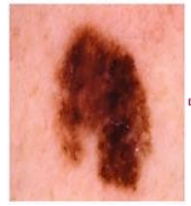

Original Resized $250 \times 250 \times 3$

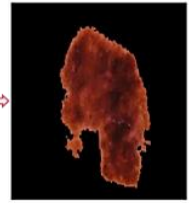

Segmented Resized Image
$250 \times 250 \times 3$

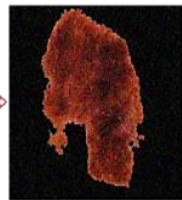

Salt \& Paper noise

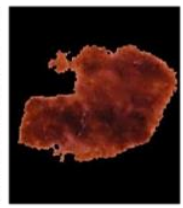

Rotation Right $90^{\circ}$

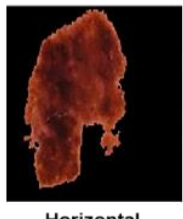
Horizontal
Flipping

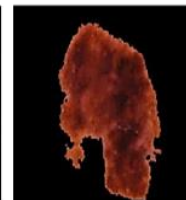
Right-Bottom
(RB)Crop, 224 X 224

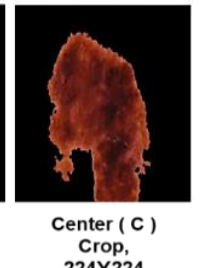

Crop,
$224 \times 224$

Figure 5. Data augmentation process

\subsection{Automatic feature extraction utilizing CNN models}

Previously, specific CNN-driven architectures extracted features that were then merged using a dense network into a fully connected layer. CNN models are among the most widely used popular 
architectures, and they have demonstrated remarkable success in medical applications. These attributes are beneficial for classification activities because they reflect various characteristics of features such as visibility, roundness of lesion, and shrink. AlexNet, VGG19, ResNet50, and GoogleNet are four successful and recent $\mathrm{CNN}$ architectures that are being considered as feature extractors for skin cancer classification. Table 1 provides a summary of the pre-trained Resnet-50, Alexnet, Googlenet and VGG19. The next subsections offer a detailed overview of certain chosen architectures. Figure 6 shows a block diagram for the proposed method.

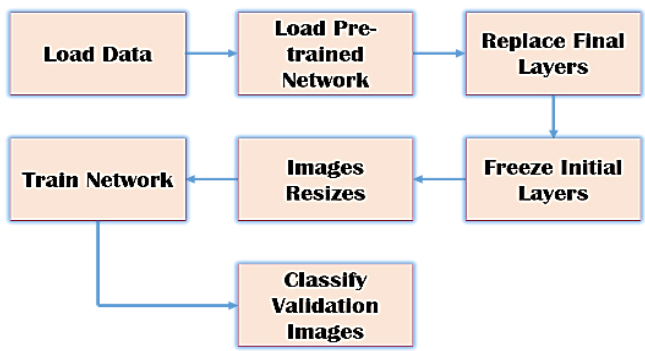

Figure 6. Workflow of CNN-based systems

Table 1. Provides a summary of the pre-trained Resnet-50, Alexnet, Googlenet and VGG19

\begin{tabular}{lccccc}
\hline & \multicolumn{5}{c}{ CNN Model description } \\
CNN model & I/P & \#Layers & \#CL & \#FC & Top-1/top-5 Error(\%) \\
\hline AlexNet & $227 \times 227$ & 8 & 5 & 3 & $41.00 / 18.00$ \\
ResNet-50 & $224 \times 224$ & 50 & 49 & 1 & $22.85 / 6.71$ \\
GoogleNet & $224 \times 224$ & 22 & 21 & 1 & $29.81 / 10.04$ \\
VGG19 & $224 \times 224$ & 19 & 16 & 3 & $27.30 / 9.00$ \\
\hline
\end{tabular}

In Table 1, the symbol "\#" denotes the number, I/P denotes the input image size, FC denotes fully connected, and CL denotes convolutional layers. AlexNet is composed of three fully linked layers and five convolutional layers. More information about AlexNet and can be found in [38]. Figure 7 shows the complex architecture of AlexNet.

ResNet is an abbreviation for Residual Network. ResNet-50 is a network that addresses the issue of accuracy degradation as network depth increases, allowing accuracy to become saturated and quickly degrading. It's a more robust 50 layers network that can identify 1000 objects in a single iteration. This network avoids the vanishing gradient issue that plagues other deep networks, making the associated features and classification challenge difficult to optimize. The implementation of skip net connections, where input is often applied to the outcome of the network's convolution blocks, is the power of ResNet-50 [39]. Figure 8 shows the complex architecture of ResNet-50.

VGG19 is similar to AlexNet but it also has more convolutional layers for image recognition. VGG19 is a deep CNN model with 19 layers with three linked layers at different levels and a pooling layer. This model has historically played a key role in image classification and localization, thanks to the help of bounding box architectures. It employs a convolutional filter with a size of $3 \times 3$ and a pooling layer network with a size of $2 \times 2$. Figure 9 depicts the complex architecture of VGG19.

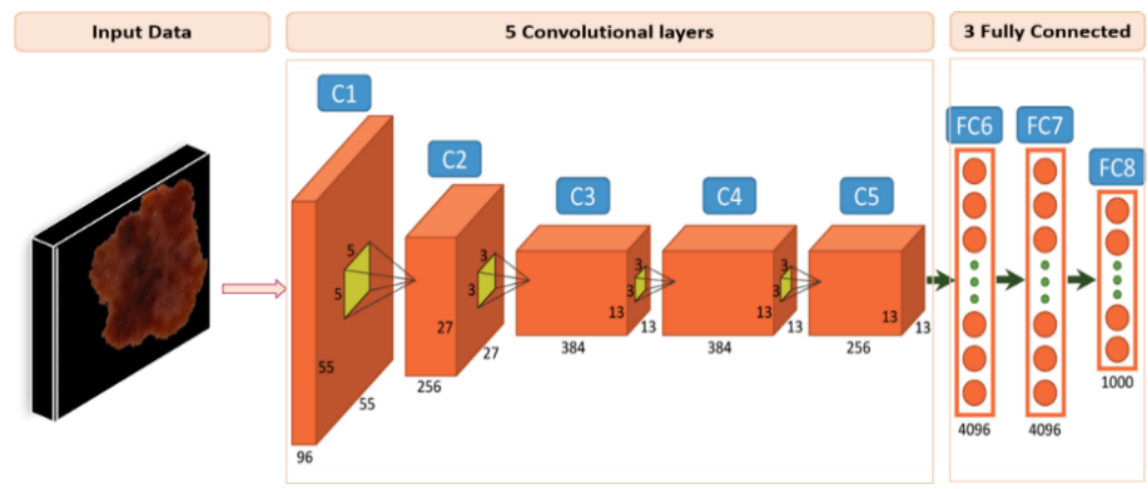

Figure 7. AlexNet architecture 


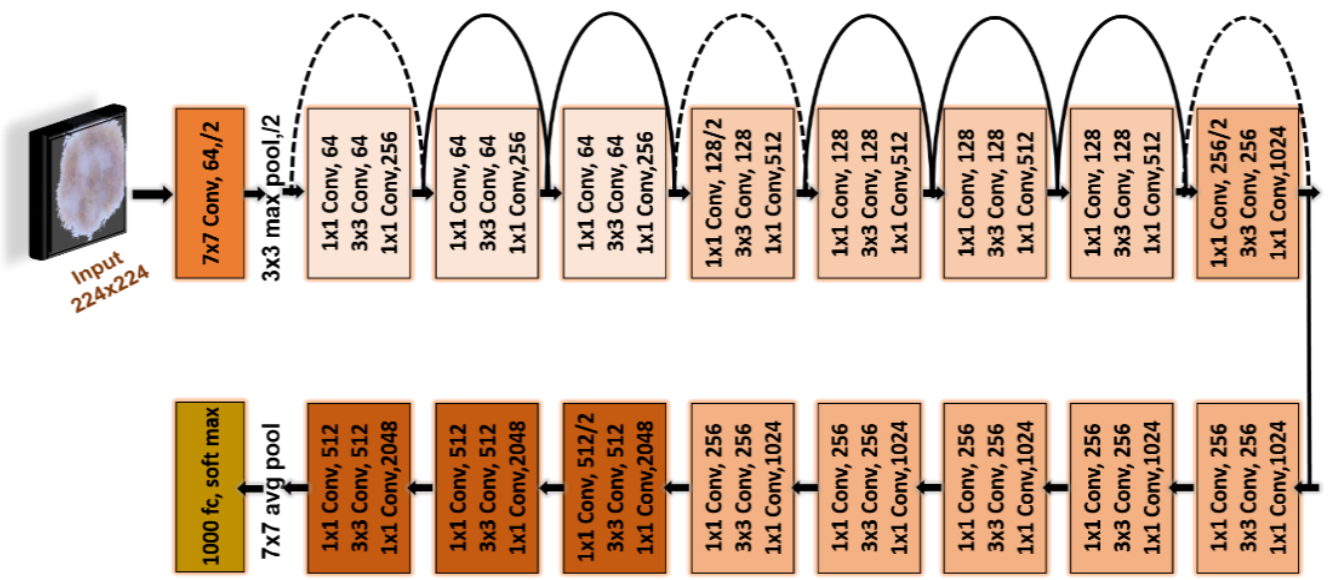

Figure 8. ResNet-50 architecture

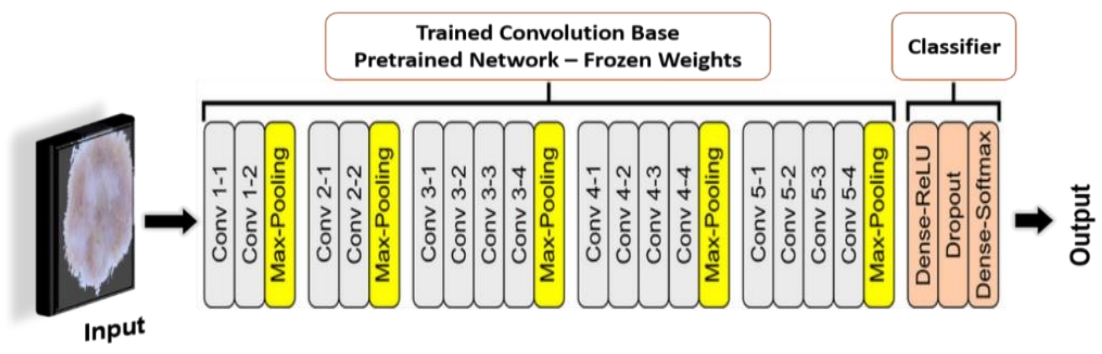

Figure 9. The personalized VGG-19 network architecture

GoogleNet is a convolutional neural network that is 22 layers deep. It was developed by Google researchers and is known as Inception Network. It's capable of pooling or convolving input data. As seen in Figure 10, the architecture consists of several inception modules stacked on top of one another. This stacking enables joint and parallel training, allowing the model to achieve quicker convergence. The benefits include quicker training and smaller scale. It does, however, have an Xception Network that will enhance the inception module's limit of divergence [40].

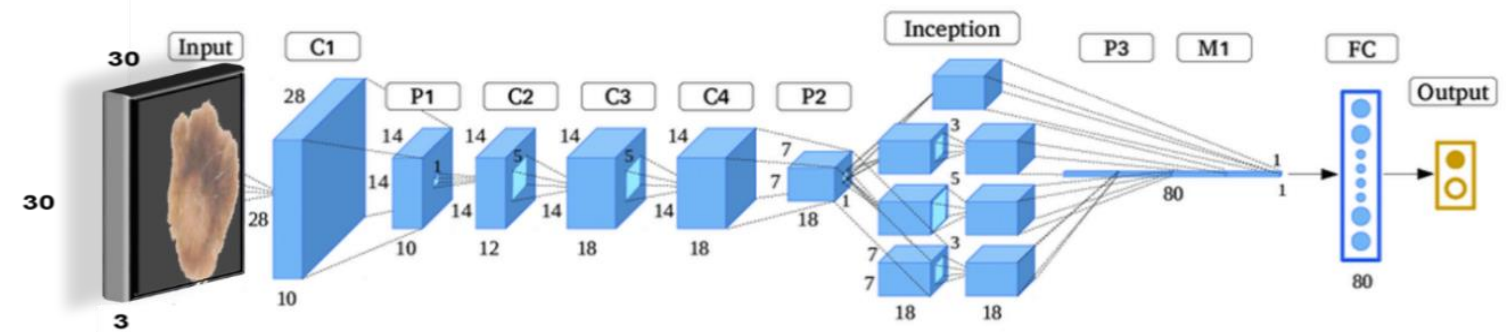

Figure 10. The customized GoogleNet network architecture

The weights of the pretrained models' convolutional layers are transferred without training to minimize training overhead, and then the completely linked layers are trained using the two datasets. Transfer learning is applied to CNN models using the final fully linked layer of the pre-trained models. The parameters of the introduced layers are trained using our ISIC and CPTAC-CM data. The vectors of activities of the last fully connected layer just before FC8 layer in AlexNet and VGG19 (i.e, the FC7 layer) or just before FC1000 in Res-Net50 or just before pool5-7x7_s1 in GoogleNet (i.e., the flatten layer), constitute the two datasets' feature descriptors. Figure 11 summarizes the proposed method. 


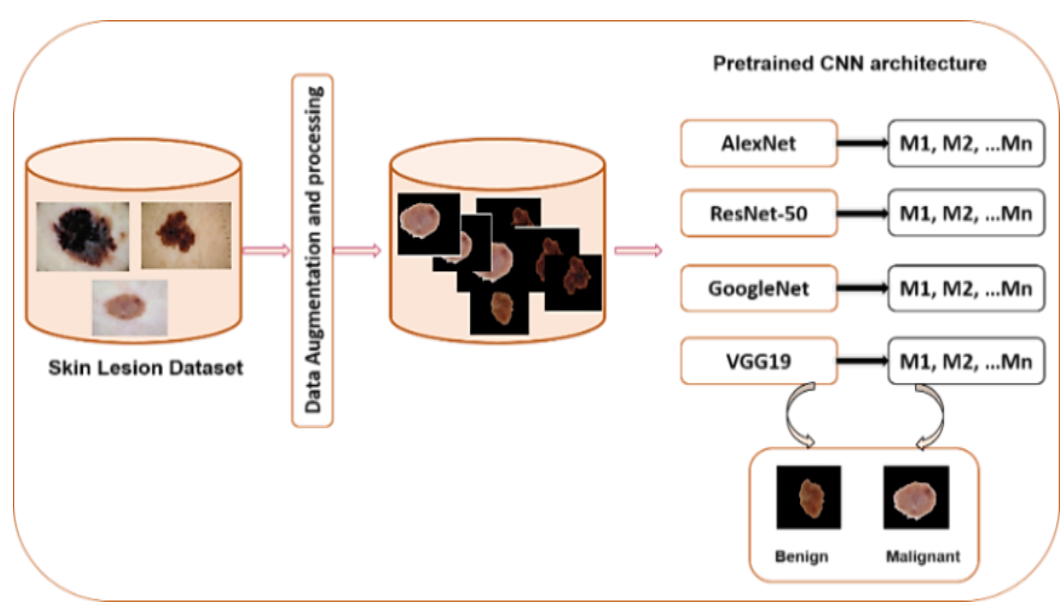

Figure 11. Block diagram of the proposed method

\subsection{Classification}

An SVM shallow classifier is utilized to categorise dermoscopic images. By breaking hyper-planes in a high-dimensional feature space, SVM aims to build a computationally efficient learning system [41]. The RoI images are classified as B or M in this phase depending on the deep extracted features. The classifier is fed with activity vectors (FC7 in AlexNet and VGG19, or the flatten layer in ResNet50, or pool5-7x7 s1 in GoogleNet), and the RoI class is output. To represent the variability of the classes, the SVM uses multi-linear mapping with a simple binary kernel. The performance is assessed for either the ResNet50 or AlexNet or VGG19 or GoogleNet, each along with either NN classifier or the SVM classifiers are dependent on standard performance evaluation metrics, such as, the classification precision must be considered, in order to choose the right model/classifier.

\subsection{Performance evaluation}

Three standard parameters are utilized to measure the efficiency of the suggested technique; accuracy (ACC.), sensitivity (SEN.), and specificity (SPE.). The evaluation metrics are described as [42]:

$$
\begin{aligned}
& \text { ACC. }=\frac{\text { No. of correct assessments }}{\text { No. of assessments }}=\frac{T P+T N}{T P+F N+F P+T N} \\
& \text { SEN. }=\frac{\text { No. of true positive assessments }}{\text { No. of positive assessments }}=\frac{T P}{T P+F N} \\
& \text { SPE. }=\frac{\text { No. of true negative assessments }}{\text { No. of negative assessments }}=\frac{T N}{T N+F P}
\end{aligned}
$$

where:

TP-true positive: a malignant image is correctly identified as malignant.

TN-true negative: a benign image is correctly identified as benign.

FP-false positive: a benign image is mistakenly diagnosed as cancerous.

FN-false negative: a malignant image is mistakenly classified as benign.

\section{EXPERIMENTAL RESULTS AND DISCUSSION}

The proposed system sensitivity, specificity and accuracy for using the SVM classifiers for skin cancer system have been determined. Two databases are used to test the proposed method. Each dataset is divided into two parts: training set and test set. The following section describes the experimental configuration, findings, and related discussions in detail.

\subsection{Experiment settings}

The system classifier is trained on $70 \%$ of the dataset and tested on $30 \%$ of the database. The Bayesian optimizer is employed in order to train the deep learning models (AlexNet, VGG19, ResNet50, and GoogleNet), by minimizing the binary cross entropy function with a learning rate of $10^{-4}$. A mini-patch size of 128 is utilised to shuffle the data through preparation. The maximum number of epochs that can be utilised is 20. The configuration values are listed in Table 2. 
Table 2. The configuration values

\begin{tabular}{lc}
\hline Configuration & Value \\
\hline Learning rate & $10^{-4}$ \\
Mini-patch size & 128 \\
Number of epochs & 20 \\
Maximum iterations & 606 (in case of alexnet), 642 (in case of resnet), 612(in case of googlenet), 600(in case of VGG19) \\
\hline
\end{tabular}

\subsection{Quantitative results}

The behavior of four CNNs as individual classifiers for melanoma identification is investigated. The best results for AlexNet and ResNet-50 CNNs, pre-trained and adapted to all datasets, are shown in Figures 12 and 13. The weight learning rate factor and the bias learn rate factor were both set to 20 in this case, with a tiny batch size of 1 .

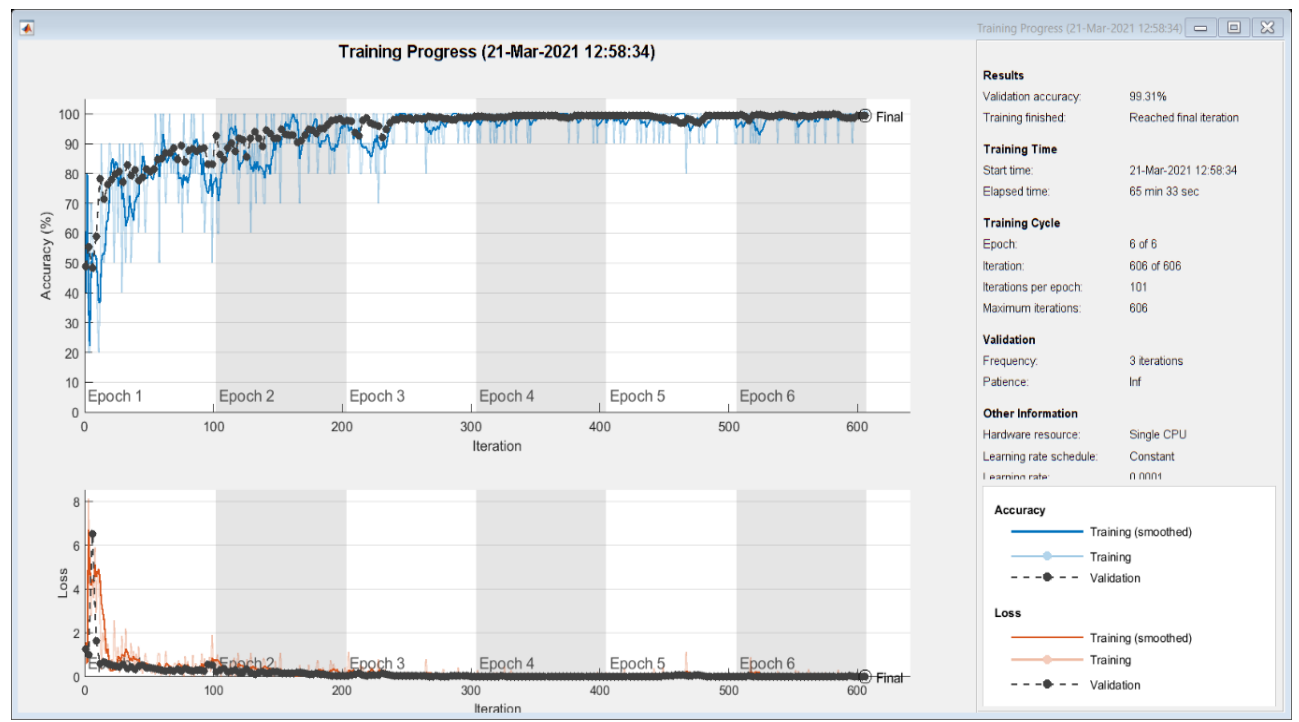

Figure 12. Training progress using AlexNet CNN for ISIC

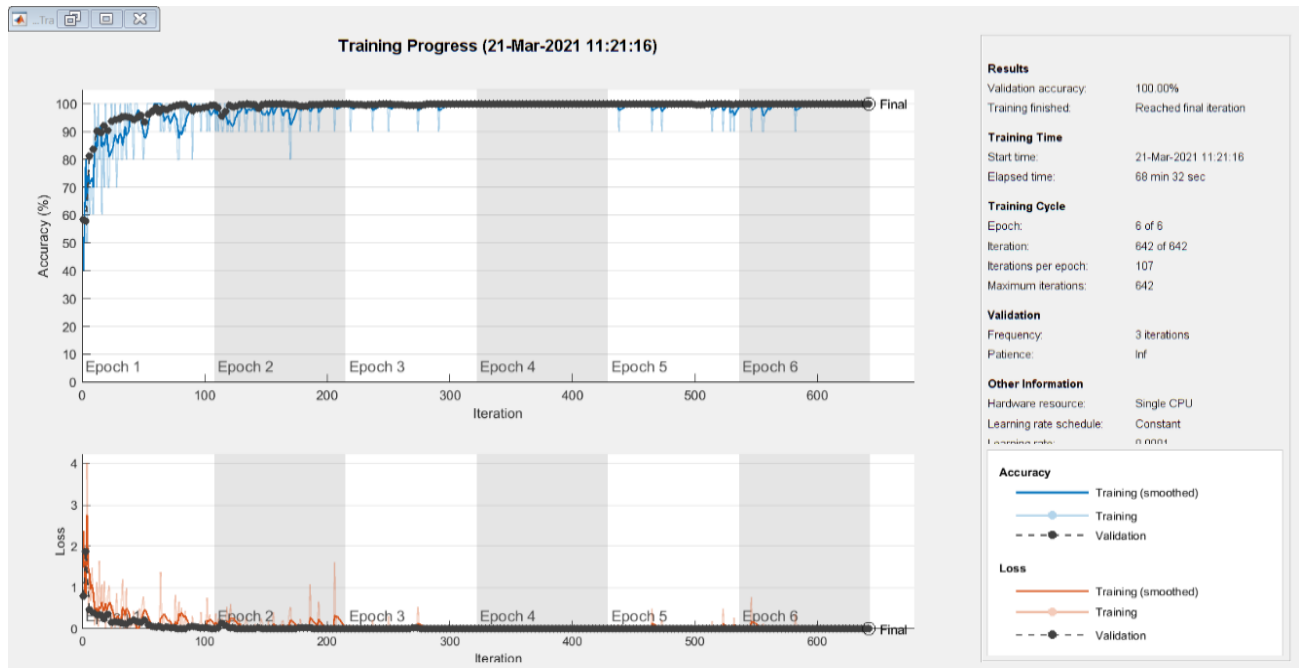

Figure 13. Training progress using ResNet-50 CNN for TCIA

As a result, some samples of validation images are shown in Figure 14 for ISIC and CPTAC-CM databases respectively. We provided the predicted labels as well as the predicted probability of the images containing certain labels for each of them. The validation accuracies were $99.3 \%$ and $99.6 \%$, respectively, in case of ResNet-50. In the global decision-fusion system, this precision would be the related weight for the 
AlexNet classifier. Figures 15-18 show the confusion matrix to categorise the two class labels utilizing CNNs models and balanced data utilizing the suggested method. As seen in the figures, the overall accuracy of all classes has reached $99.8 \%$, which, as expected, outperforms the case of utilising imbalanced data. The receiver operating characteristic (ROC) curve is utilized to display the results of the proposed simulation model. Figures 19 and 20 show the ROC curves for categorizing the 2 classes utilizing AlexNet and ResNet$50 \mathrm{CNNs}$ and balanced data utilizing our proposed technique. Table 3 gives the results of the proposed system. Table 4 gives a comparison between the proposed approach and the previously suggested techniques in a brief manner.

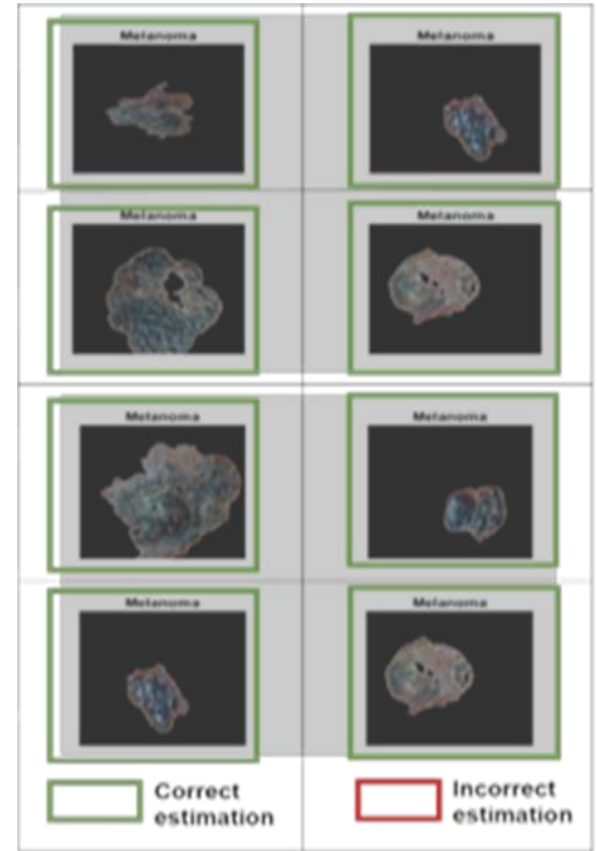

(a)

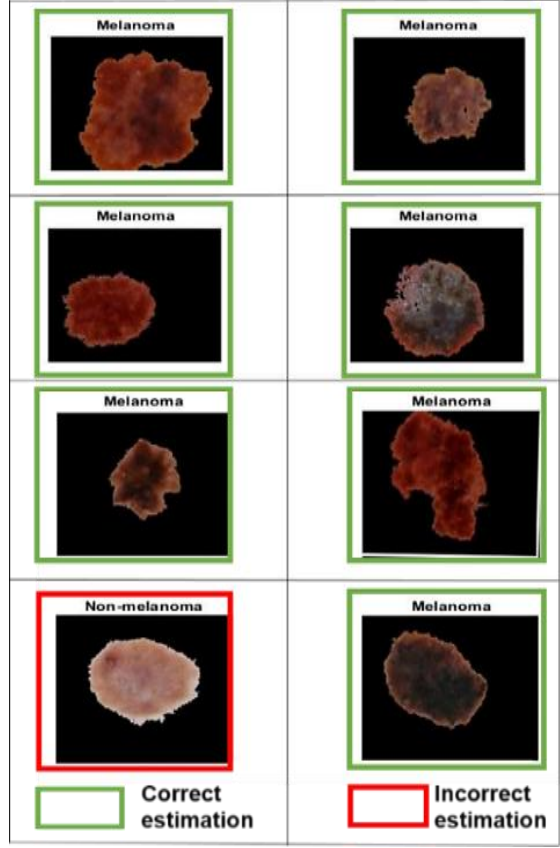

(b)

Figure 14. Validation images with foretold labels in the case of ResNet-50 for (a) CPTAC-CM datasets and (b) ISIC datasets

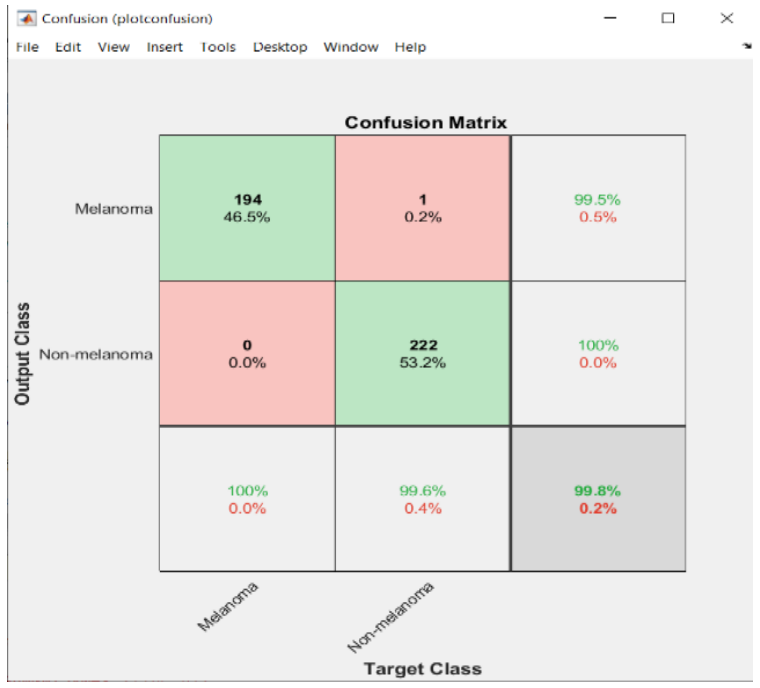

Figure 15. Confusion Matrix presenting categorization results in case of GoogleNet for ISIC dataset

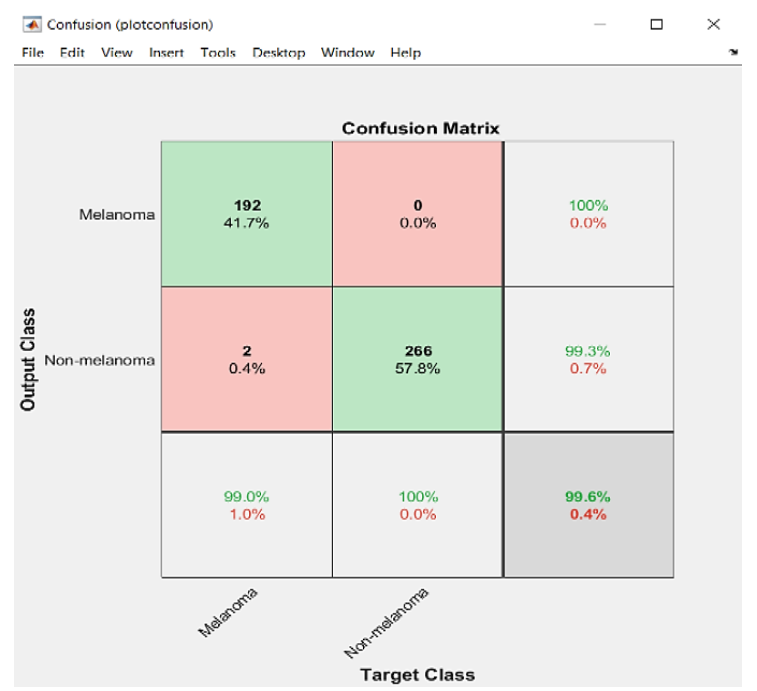

Figure 16. Confusion Matrix presenting categorization results in case of VGG19 for ISIC dataset 


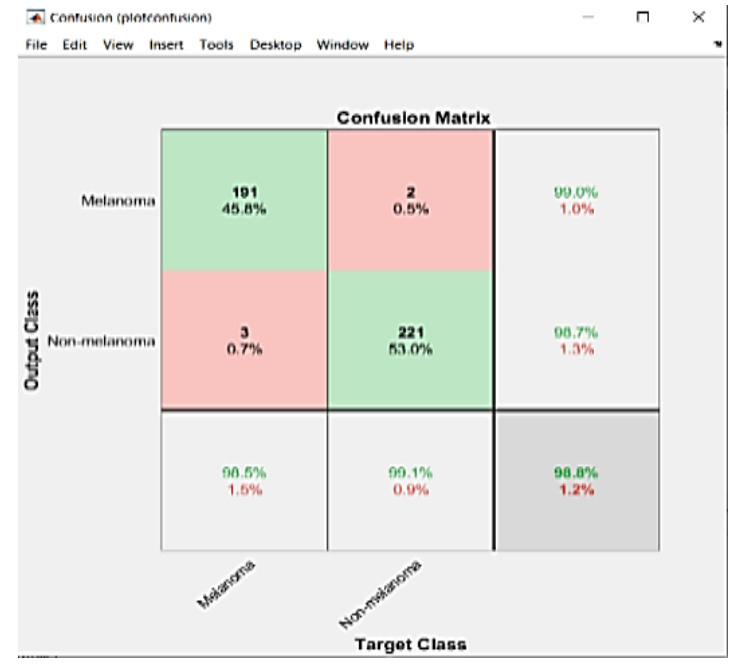

Figure 17. Confusion Matrix presenting categorization results in case of AlexNet for ISIC dataset

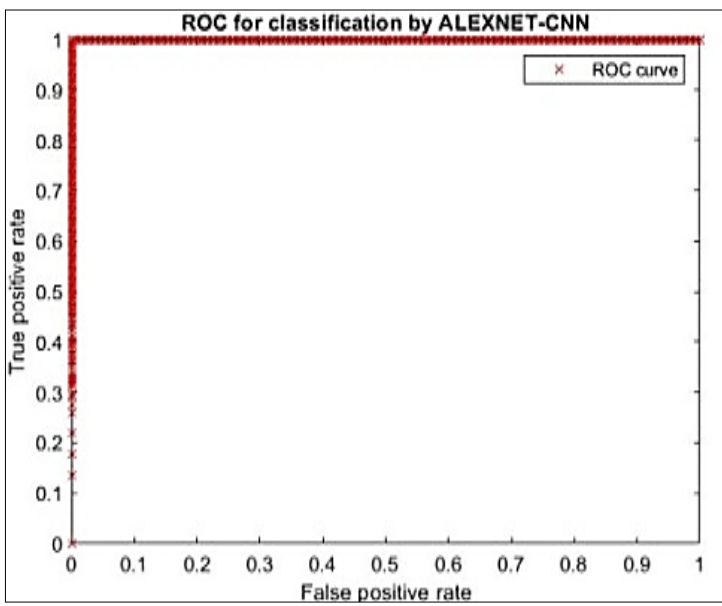

Figure 19. ROC curves in skin lesions classification of ISIC 2019 (Plot of TPR vs FPR for two distinct diagnostic categories: Melanoma, Non-Melanoma) AlexNet CNN for ISIC

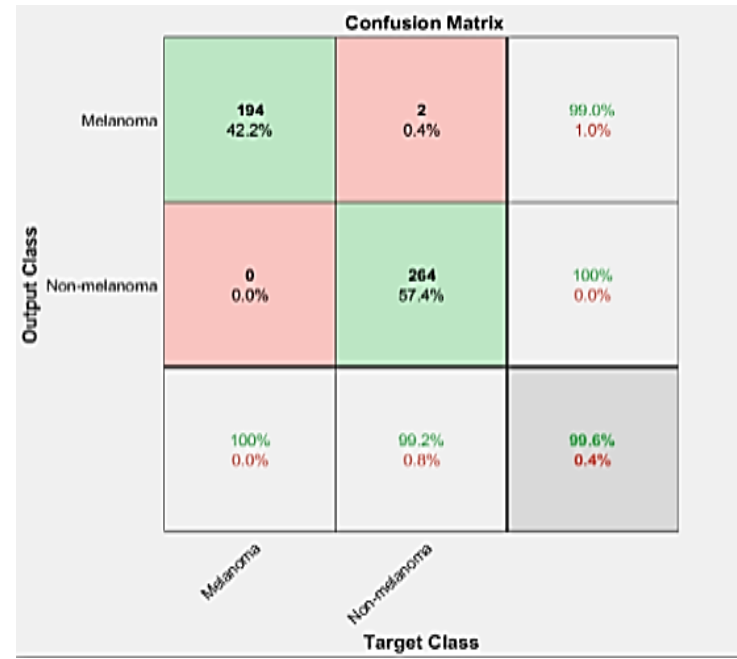

Figure 18. Confusion Matrix presenting categorization results in case of ResNet-50 for ISIC dataset

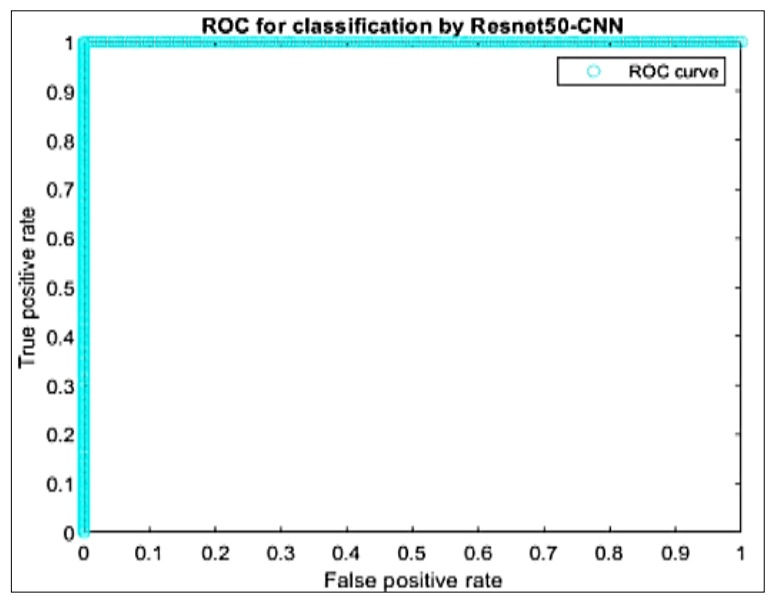

Figure 20. ROC curves in skin lesions classification of ISIC 2019 (Plot of TPR vs FPR for two distinct diagnostic categories: Melanoma, Non-Melanoma) ResNet-50 CNN for ISIC

\subsection{Discussion}

Table 3 shows the accuracies, sensitivities, and specificities of the different training data setting of the proposed system. Where proposed method 1 corresponds to ISIC dataset with data augmentation, and proposed method 2 corresponds to CPTAC-CM dataset with data augmentation. We utilized CNNs that had been pre-trained with a huge image dataset to detect skin lesions. The previously explained four models are applied and compared for each case. As shown Table 3 demonstrates that using AlexNet and GoogleNet with a SVM classifier achieves the highest performance in all examined cases. These results highlight the potentiality of using AlexNet/SVM and GoogleNet/SVM, taking into account augmentation, for achieving high performance, the mean accuracies are $99.9 \%$ and $99.8 \%$ respectively. Utilizing transfer learning, the CNNs were able to classify skin lesions successfully. The best findings were achieved utilizing AlexNet and GoogleNet pre-trained with the two datasets. In comparison with previous works, shown in Table 4, the presented model had proven high accuracy in early detection of skin cancer. This demonstrates the approach's capability to detect melanoma at an early stage. When comparing the findings of several research, as shown in Table 4, to the results of the present study, our technique performed even better by using 4 deep neural networks. 
Table 3. Proposed system for different training data using four deep learning models (Resnet50, Alexnet, Googlenet And Vgg19) With SVM Classifier and transfer learning classifier and using two datasets

\begin{tabular}{lllllllll} 
& & \multicolumn{3}{c}{ SVM Classifier } & \multicolumn{4}{c}{ Transfer Learning } \\
\hline Case & Dataset & Method & ACC. \% & SEN.\% & SPE. \% & ACC. \% & SEN.\% & SPE. \% \\
Case I & ISIC & ResNet50 & $99.3 \%$ & $99.5 \%$ & $99.1 \%$ & $99.5 \%$ & $99.5 \%$ & $99.6 \%$ \\
& CPTAC-CM & ResNet50 & $99.6 \%$ & $99 \%$ & $100 \%$ & $\mathbf{1 0 0 \%}$ & $\mathbf{1 0 0 \%}$ & $\mathbf{1 0 0 \%}$ \\
Case II & ISIC & VGG19 & $98.8 \%$ & $98.5 \%$ & $99.1 \%$ & $98.9 \%$ & $98.6 \%$ & $99.2 \%$ \\
& CPTAC-CM & VGG19 & $99.6 \%$ & $100 \%$ & $99.3 \%$ & $99.8 \%$ & $99.5 \%$ & $100 \%$ \\
Case IV & ISIC & GoogleNet & $\mathbf{9 9 . 8 \%}$ & $\mathbf{9 9 . 5 \%}$ & $\mathbf{1 0 0 \%}$ & $\mathbf{9 9 . 6 \%}$ & $\mathbf{9 9 . 8 \%}$ & $\mathbf{1 0 0 \%}$ \\
& CPTAC-CM & GoogleNet & $99.87 \%$ & $99.5 \%$ & $100 \%$ & $99.9 \%$ & $100 \%$ & $100 \%$ \\
Case V & ISIC & AlexNet & $98.8 \%$ & $99 \%$ & $98.7 \%$ & $99.31 \%$ & $99.54 \%$ & $99.05 \%$ \\
& CPTAC-CM & AlexNet & $\mathbf{9 9 . 9 \%}$ & $\mathbf{1 0 0 \%}$ & $\mathbf{9 9 . 6 \%}$ & $\mathbf{1 0 0 \%}$ & $\mathbf{1 0 0 \%}$ & $\mathbf{1 0 0 \%}$ \\
\hline
\end{tabular}

Table 4. Comparison between related methods and proposed models

\begin{tabular}{|c|c|c|c|c|}
\hline References & Dataset & Method & Observations & Accuracy $\%$ \\
\hline Li and Shen [30] & ISIC & Two deep learning method & lesion classification & $91.2 \%$ \\
\hline Hosny et al. [32] & ISIC2018 & $\begin{array}{l}\text { AlexNet, 10-fold cross-validation, fine-tune, } \\
\text { transfer learning, Augmentation, GPU }\end{array}$ & lesion classification & $92.99 \%$ \\
\hline Hosny et al. [33] & ISIC2019 & $\begin{array}{l}\text { GoogleNet, Similarity score, bootstrap weighted } \\
\text { SVM classifier, SoftMax, fine-tune, transfer } \\
\text { learning, Augmentation. }\end{array}$ & lesion classification & $98.70 \%$ \\
\hline \multirow[t]{4}{*}{ Khan et al. [34] } & ISBI 2016 & Novel Deep Saliency Segmentation method, ten- & \multirow[t]{4}{*}{ lesion classification } & $95.38 \%$ \\
\hline & ISBI 2017 & layer convolutional neural network $(\mathrm{CNN})$, and & & $95.79 \%$ \\
\hline & ISBI 2018 & Kernel Extreme Learning Machine (KELM) & & $92.69 \%$ \\
\hline & $\mathrm{PH} 2$ & classifier. & & $98.70 \%$ \\
\hline $\begin{array}{l}\text { Abayomi-Alli } \\
\text { et al. }[35]\end{array}$ & $\mathrm{PH} 2$ & $\begin{array}{l}\text { Deep CNN-based using SqueezeNet and } \\
\text { covariant SMOTE augmentation in low } \\
\text { dimensional manifold. }\end{array}$ & lesion classification & $92.18 \%$ \\
\hline Kawahara et al. [43] & ISIC & Linear classifier CNN deep learning. & lesion classification & $85.5 \%$ \\
\hline Codella et al. [44] & ISIC & Deep learning, sparse coding, SVM. & lesion classification & $93.1 \%$ \\
\hline \multirow[t]{4}{*}{ Proposed Method 1} & \multirow[t]{4}{*}{ ISIC } & CNNs based on GoogleNet. & lesion classification & $99.8 \%$ \\
\hline & & CNNs based on AlexNet. & lesion classification & $98.8 \%$ \\
\hline & & CNNs based on ResNet-50. & lesion classification & $99.3 \%$ \\
\hline & & CNNs based on VGG19. & lesion classification & $98.8 \%$ \\
\hline \multirow[t]{4}{*}{ Proposed Method 2} & \multirow{4}{*}{$\begin{array}{l}\text { CPTAC- } \\
\text { CM }\end{array}$} & CNNs based on GoogleNet. & lesion classification & $99.87 \%$ \\
\hline & & CNNs based on AlexNet. & lesion classification & $99.9 \%$ \\
\hline & & CNNs based on ResNet- 50 . & lesion classification & $99.6 \%$ \\
\hline & & CNNs based on VGG19. & lesion classification & $99.6 \%$ \\
\hline
\end{tabular}

\subsubsection{Limitations}

The color and texture of different types of skin lesions differ only slightly. Diverse images may result in images of skin lesions that do not belong to that specific lesion category. Another issue was the existence of certain dermatologist-drawn marks close to the lesions, as well as the existence of drops of water. Despite producing promising results in comparison to competing techniques, the proposed method fails to correctly classify all of the images. There are some images in Figure 14 that depict examples of erroneous classification. In the future, it is intended to add more features and different deep learning algorithms to enhance the accuracy of classification.

\section{CONCLUSION}

In this paper, a CAD system for early detection of skin cancer was presented based on deep learning. The CNN models utilized extracted features from RoI in order to enhance the deep learning model training capabilities. A SVM classifier was utilized to categorise dermoscopic images into two classes; benign or malignant. Experimental results using four CNN models on two different datasets had confirmed the superiority of the proposed methods over previous work. Results have shown that, under all conditions, the AlexNet and ResNet-50 frameworks were superior to other models with an average accuracy of $99.9 \%$ and $100 \%$ respectively. These results confirm that the proposed technique can be utilized as an effective diagnostic tool for early diagnosis of melanoma. Future work will focus on multi-classification and grading of different types of skin cancer. Another challenge is to reduce computational time and to use different datasets with larger number of images.

\section{REFERENCE}

[1] D. C. Grossman et al., "Behavioral Counseling to Prevent Skin Cancer: US Preventive Services Task Force Recommendation Statement," JAMA-Journal of the American Medical Association, vol. 319, no. 11, pp. 1134-1142, 2018, doi: 10.1001/jama.2018.1623. 
[2] R. T. Al-Jamal et al., "The Pediatric Choroidal and Ciliary Body Melanoma Study a Survey by the European Ophthalmic Oncology Group," Ophthalmology, vol. 123, no. 4, pp. 898-907, 2016, doi: 10.1016/j.ophtha.2015.12.024

[3] N. Codella, J. Cai, M. Abedini, R. Garnavi, A. Halpern, and J. R. Smith, "Deep Learning, Sparse Coding, and SVM for Melanoma Recognition in Dermoscopy Images," International Workshop on Machine Learning in Medical Imaging, vol. 9352, pp. 118-126, October. 2015, doi: 10.1007/978-3-319-24888-2.

[4] F. Dalila, A. Zohra, K. Reda, and C. Hocine, "Segmentation and Classification of Melanoma and Benign Skin Lesions," Optik International Journal for Light and Electron Optics, vol. 140, pp. 749-761, 2017, doi: 10.1016/j.ijleo.2017.04.084.

[5] N. Razmjooy, B. S. Mousavi, F. Soleymani, and M. H. Khotbesara, "A Computer-Aided Diagnosis System for Malignant Melanomas," Neural Comput Appl, vol. 23, pp. 2059-2071, 2013, doi: 10.1007/s00521-012-1149-1.

[6] N. Razmjooy, F. R. Sheykhahmad, and N. Ghadimi, "A hybrid neural network-world cup optimization algorithm for melanoma detection," Open Medicine, vol. 13, no. 1, pp. 9-16, 2018, doi: 10.1515/med-2018-0002.

[7] M. Silveira et al., "Comparison of Segmentation Methods for Melanoma Diagnosis in Dermoscopy Images," IEEE Journal of Selected Topics in Signal Processing, vol. 3, no. 1, pp. 35-45, 2009, doi: 10.1109/JSTSP.2008.2011119.

[8] R. L. Siegel, K. D. Miller, and A. Jemal, "Cancer Statistics, 2019,” CA: A Cancer Journal for Clinicians, 2019, doi: 10.3322/caac.21551.

[9] V. M. Cohen et al., "Staging Uveal Melanoma with Whole-Body Positron-Emission Tomography/Computed Tomography and Abdominal Ultrasound: Low Incidence of Metastatic Disease, High Incidence of Second Primary Cancers," Middle East African Journal of Ophthalmology; vol. 25, no. 2, pp. 91, 2018, doi: 10.4103/meajo.MEAJO_96_18.

[10] N. Ghadimi, "An Adaptive Neuro-Fuzzy Inference System for Islanding Detection in Wind Turbine as Distributed Generation," Complexity, Hoboken, vol. 21, no. 1, pp. 10-20, 2015, doi: 10.1002/cplx.21537.

[11] K. Atul and M. Debajyoti, "SVM Classifier Based Melanoma Image Classification," Research Journal of Pharmacy and Technology, vol. 10, no. 12, pp. 4391-4392, 2017, doi: 10.5958/0974-360X.2017.00808.3.

[12] K. Narasimhan and V. Elamaran, "Wavelet-Based Energy Features for Diagnosis of Melanoma from Dermoscopic Images," International Journal of Biomedical Engineering and Technology, vol. 20, no. 3, pp. 243-252, 2016, doi: 10.1504/IJBET.2016.075427.

[13] Y. Ali and S. Hamed, "Early Breast Cancer Detection Using Mammogram Images: a Review Of Image Processing Techniques," Biosciences Biotechnology Research Asia, vol. 12, no. 1, pp. 225-234, 2015, doi: 10.13005/bbra/1627.

[14] N. Razmjooy, B. S. Mousavi, and F. Soleymani, "A Real-Time Mathematical Computer Method for Potato Inspection Using Machine Vision," Computers \& Mathematics with Applications, vol. 63, no. 1, pp. 268-279, 2012, doi: 10.1016/j.camwa.2011.11.019.

[15] N. Razmjooy, B. S. Mousavi, and F. Soleymani, "A Hybrid Neural Network Imperialist Competitive Algorithm for Skin Color Segmentation," Mathematical and Computer Modelling, vol. 57, no. 3-4, pp. 848-856, 2013, doi: 10.1016/j.mcm.2012.09.013.

[16] P. Moallem and N. Razmjooy, "A Multi Layer Perceptron Neural Network Trained by Invasive Weed Optimization for Potato Color Image Segmentation," Trends in Applied Sciences Research, vol. 7, no. 6, pp. 445-455, 2012, doi: 10.3923/tasr.2012.445.455.

[17] S. Mirjalili, "Genetic Algorithm: in Evolutionary Algorithms and Neural Networks," Springer, Cham, pp. 43-55, 2019, doi: 10.1007/978-3-319-93025-1_4.

[18] F. P. Such, V. Madhavan, E. Conti, J. Lehman, K. O. Stanley, and J. Clune, "Deep Neuroevolution: Genetic Algorithms are a Competitive Alternative for Training Deep Neural Networks for Reinforcement Learning," Neural and Evolutionary Computing (cs.NE); Machine Learning (cs.LG), arXiv preprint arXiv:1712.06567, 2018.

[19] O. Abedinia, N. Amjady, and N. Ghadimi, "Solar Energy Forecasting Based on Hybrid Neural Network and Improved Metaheuristic Algorithm," Wiley Online Library: Computational Intelligence, vol. 34, no. 1, pp. 241-260, 2018, doi: 10.1111/coin.12145.

[20] N. Ghadimi, A. Akbarimajd, H. Shayeghi, and O. Abedinia, "Two Stage Forecast Engine with Feature Selection Technique and Improved Meta-Heuristic Algorithm for Electricity Load," Forecasting Energy, vol. 161, pp. 130-142, 2018, doi: 10.1016/j.energy.2018.07.088.

[21] N. Razmjooy and M. Ramezani, "Training Wavelet Neural Networks Using Hybrid Particle Swarm Optimization and Gravitational Search Algorithm for System Identification," International Journal of Mechatronics, Electrical and Computer Technology, vol. 6, no. 21, pp. 2987-2997, 2016, doi: 649123/10215.

[22] N. Ghadimi, “A New Hybrid Algorithm Based on Optimal Fuzzy Controller in Multimachine Power System,” Complexity, vol. 21, no. 1, pp. 78-93, 2015, doi: 10.1002/cplx.21544.

[23] A. R. Jadhav, A. G. Ghontale, and V. K. Shrivastava, "Segmentation and Border Detection of Melanoma Lesions Using Convolutional Neural Network and SVM," In Computational Intelligence: Theories, Applications and Future Directions Volume I. Advances in Intelligent Systems and Computing, vol. 798, 2019, doi: 10.1007/978-981-13-1132-1_8.

[24] O. Ronneberger, P. Fischer, and T. Brox, "Convolutional Networks for Biomedical Image Segmentation," Medical Image Computing and Computer-Assisted Intervention (MICCAI), Springer, LNCS, vol. 9351, pp. 234-241, 2015, doi: 10.1007/978-3319-24574-4_28.

[25] A. Hamidinekoo, E. Denton, A. Rampun, K. Honnor, and R. Zwiggelaar, "Deep Learning in Mammography and Breast Histology, An Overview and Future Trends," Medical image analysis, vol. 47, pp. 45-67, 2018, doi: 10.1016/j.media.2018.03.006.

[26] P. Tschandl, C. Rosendahl, and H. Kittler, "The HAM10000 dataset, a large collection of multi-source dermatoscopic images of common pigmented skin lesions," Scientific data, vol. 5, pp. 180161-180169, 2018, doi: 10.1038/sdata.2018.161.

[27] N. C. F. Codella et al., "Skin lesion analysis toward melanoma detection: A challenge at the 2017 international symposium on biomedical imaging (isbi), hosted by the international skin imaging collaboration (isic)," In 2018 IEEE 15th international symposium on biomedical imaging, pp. 168-172, IEEE 2018, arXiv:1710.05006.

[28] M. Combalia et al., "BCN20000: Dermoscopic Lesions in the Wild," arXiv:1908.02288, 2019.

[29] R. S. Lee, F. Gimenez, A. Hoogi, and D. Rubin, "The Cancer Imaging Archive,” vol. 8, 2016.

[30] Y. Li and L. Shen, "Skin Lesion Analysis Towards Melanoma Detection Using Deep Learning Network," Sensors, vol. 18, 2018, doi: 10.3390/s18020556.

[31] M. Kassem, K. M. Hosny, R. Damasevicius, and M. M. Eltoukhy, "Machine Learning and Deep Learning Methods for Skin Lesion Classification and Diagnosis: A Systematic Review," Diagnostics, vol. 11, no. 8, 2021, doi: 10.3390/diagnostics11081390.

[32] K. M. Hosny, M. A. Kassem, and M. M. Foaud, "Classification of Skin Lesions into Seven Classes Using Transfer Learning with AlexNet," Journal of Digital Imaging, vol. 33, pp. 1325-1334, 2020, doi: 10.1007/s10278-020-00371-9.

[33] K. M. Hosny, M. A. Kassem, and M. M. Foaud, "Skin Lesions Classification into Eight Classes for ISIC 2019 Using Deep Convolutional Neural Network and Transfer learning," IEEE Access, vol. 8, pp. 114822-114832, 2020, doi: 10.1109/ACCESS.2020.3003890. 
[34] M. A. Khan, M. Sharif, T. Akram, R. Damasevicius, and R. Maskeliunas, "Skin Lesion Segmentation and Multiclass Classification Using Deep Learning Features and Improved Moth Flame Optimization,” Diagnostics, vol. 11, no. 5, p. 811, 2021, doi: 10.3390/diagnostics11050811.

[35] O. Abayomi-Alli, R. Damasevicius, S. Misra, R. Maskeliunas, and A. Abayomi-Alli, "Malignant Skin Melanoma Detection Using Image Augmentation by Oversampling in Nonlinear Lower-Dimensional Embedding Manifold," Turkish Journal of Electrical Engineering and Computer Sciences, vol. 29, pp. 2600-2614, 2021, doi: 10.3906/elk-2101-133.

[36] J. A. Jaleel, S. Salim, and R. B. Aswin, "Computer Aided Detection of Skin Cancer," International Conference on Circuits, Power and Computing Technologies (ICCPCT), IEEE, Conference Location: Nagercoil, India, 2013, pp. 1137-1142, doi: 10.1109/iccpct.2013.6529066.

[37] A. Kori, S. S. Chennamsetty, and V. Alex, "Ensemble of Convolutional Neural Networks for Automatic Grading of Diabetic Retinopathy and Macular Edema," Computer Science, Computer Vision and Pattern Recognition, arXiv preprint arXiv, pp. 1809$0422,2018$.

[38] A. Krizhevsky, I. Sutskever, and G. E. Hinton, "ImageNet Classification with Deep Convolutional Neural Networks," Advances in neural information processing systems, vol. 25, no. 2, pp. 1097-1105, 2012, doi:10.1145/3065386.

[39] E. Rezende, G. Ruppert, T. Carvalho, F. Ramos, and P. De Geus, "Malicious software classification using transfer learning of resnet-50 deep neural network," 16th IEEE International Conference on Machine Learning and Applications (ICMLA) at: Cancun, Mexico, IEEE, pp. 1011-1014, 2017, doi: 10.1109/ICMLA.2017.00-19.

[40] C. Szegedy, V. Vanhoucke, S. Ioffe, J. Shlens, and Z. Wojna, "Rethinking the Inception Architecture for Computer Vision," Computer Science - Computer Vision and Pattern Recognition, 2016, doi: 10.1109/CVPR.2016.308.

[41] D. A. Pisner and D. M. Schnyer, "Support vector machine," in Machine Learning, ed: Elsevier, pp. 101-121, 2020, doi: 10.1016/B978-0-12-815739-8.00006-7.

[42] W. Zhu, N. Zeng, and N. Wang, "Sensitivity, specificity, accuracy, associated confidence interval and ROC analysis with practical SAS implementations," NESUG proceedings: Health Care and Life Sciences, Baltimore, Maryland., vol. 19, p. $67,2010$.

[43] J. Kawahara, A. BenTaieb, G. Hamarneh, "Deep Features to Classify Skin Lesions," 2016 IEEE 13th International Symposium on Biomedical Imaging (ISBI), Prague, Czec h Republic, pp. 1397-1400, 2016, doi: 10.1109/ISBI.2016.7493528.

[44] N. Codella, J. Cai, M. Abedini, R. Garnavi, A. Halpern, and J.R. Smith, "Deep learning, sparse coding, and SVM for melanoma recognition in dermoscopy images," In Proceedings of the 6th International Workshop on Machine Learning in Medical Imaging, Munich, Germany, vol. 9352, pp. 118-126, 2015, doi: 10.1007/978-3-319-24888-2_15.

\section{BIOGRAPHIES OF AUTHORS}
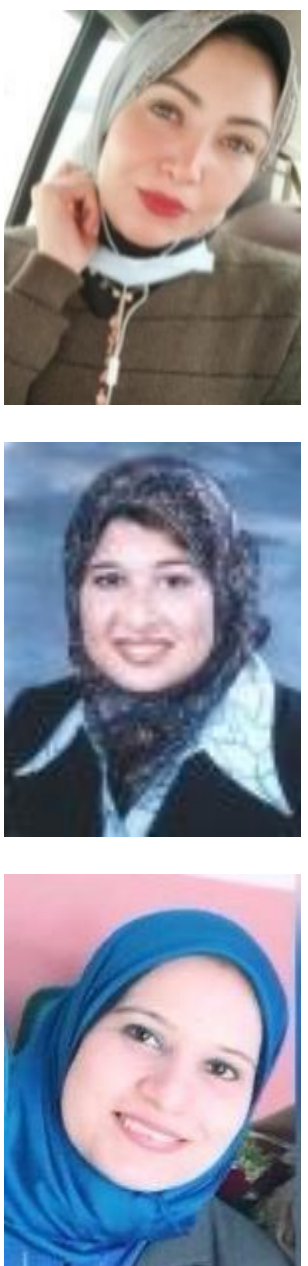

Amal G. Diab (D) SC SC received the B.Sc. degree in communication engineering from the Department of Communications and Electronics Engineering, MISR Higher Institute for Engineering and Technology (MET), Mansoura, Egypt. She is currently pursuing the M.Sc. degree in communication engineering from the Department of Electronics and Communications Engineering, Faculty of Engineering, Mansoura University. Her major research interests include image processing, medical imaging, and machine learning. She can be contacted at email: galalama1577@gmail.com.

Nehal Fayez (iD) If SC $\mathrm{P}$ received the B.Sc. degree in electronics and communications from the Faculty of Engineering, Mansoura University, in May 2000, and the M.Sc. and Ph.D. degrees in electrical communications from the Faculty of Engineering, Mansoura University, in 2003 and 2008, respectively. She was appointed as a Professor in September 2018. Microwave and antenna research, optical fibres, and plasmonic models are among her research interests. She is an IEEE Communication Society and OSA member. In 2001, she received the Egyptian Society of Engineers' Scientific Excellence Award. In July 2018, she received the State Incentive Award from the Academy of Scientific Research. She can be contacted at email: nfayez@zewailcity.edu.eg.

Mervat Mohamed El-Seddek (iD) SIS SC received the B.Sc. degree in electronics and communications from the Electronic and Communication Department, Faculty of Engineering, Mansoura University, in 1999, and the M.Sc. and Ph.D. degrees in electrical communications from the Faculty of Engineering, Mansoura University, in 2009 and 2015, respectively. She was appointed as an Assistant Professor in the Department of Communications and Electronics Engineering at the Mansoura Higher Institute of Engineering and Technology. Image processing, medical imaging, and machine learning are among the primary research areas. Member at IEEE. She can be contacted at email: mervat.elseddek@ ieee.org. 